\title{
Could Versus Should: A Reply to Sammons ${ }^{1}$
}

\author{
Kenneth M. Adams ${ }^{2,3}$ and Linas A. Bieliauskas ${ }^{2}$
}

Sammons (1994) believes that our critique of organized psychology's efforts to gain limited prescriptive privileges (Adams \& Bieliauskas, 1994) is comprised of pseudo-problems (e.g., the noncurative nature of psychopharmacology) or issues that can readily be handled (e.g., malpractice exposure). We disagree and attempt herein to inject some reality into the picture of the bright new world of psychologists armed with prescription pads. Most importantly, the seemingly vanished distinction between what could be done politically or legislatively and what should be done for the profession and the public good is redrawn.

KEY WORDS: prescription, drug; psychopharmacology; psychology, clinical; psychology, medical; credentialing; malpractice; legislation, pharmacy.

\section{INTRODUCTION}

Sammons (1994; preceding paper) has responded to our report (Adams \& Bieliauskas, 1994; this issue), concluding that we have not "proved" why psychologists should not move, posthaste, to gain limited prescriptive privileges. Using this logic, we probably would also fail to convince our colleague that forensic psychologists should not be junior lawyers or that neuropsychologists should not be cryptotoxicologists. The focus here should not be what we might pull off legislatively if we try, but the reasons

\footnotetext{
${ }^{1}$ This paper does not reflect the official policy of the Department of Veterans Affairs or the University of Michigan.

${ }^{2}$ Departments of Psychiatry and Psychology, University of Michigan, and Psychology Service, VA Medical Center, Ann Arbor, Michigan.

${ }^{3}$ To whom correspondence should be addressed at Psychology Service (116B), VA Medical Center, 2215 Fuller Road, Ann Arbor, Michigan 48105-2300.
} 
why we might wish to persuade the public, their lawmakers, and regulators that prescriptive privileges for psychologists is a good idea.

There are five general tenets that Sammons advances: (1) Psychologists will do at least no worse in prescribing psychoactive medications than psychiatrists or physicians in general, and psychologists' better appreciation of psychotherapeutic methods will serve as a disincentive to reflexive reliance upon medication; (2) medications that are not curative are of the same standing in medicine as ones that are indeed curative, and regardless of this distinction, psychologists need to get in on the action; (3) malpractice is not a worry if psychologists obtain prescription privileges; (4) the economics of the prescription initiative are above reproach; and (5) the prescriptive privilege initiative is just the latest in inevitable skirmishes between psychiatry and psychology, and psychologists continue to be on the side of the angels.

Sammons paints an optimistic picture concerning the likely success of a relatively brief program in preparing psychologists to prescribe. In fact, the current curricula and protocols that prepare physicians to prescribe psychotropic medications seem to many to be inadequate. A very substantial proportion of those prescriptions written for psychotropic medicines is not written by psychiatrists. In this regard, organized medicine has erred in its traditional assumption that every physician has, quod erat demonstrandum, an understanding of all facets of health care. We would do well to observe this problem in medicine with humility and a desire to learn from it, instead of diving into it for our own profit.

The American College of Neuropsychopharmacology (ACNP) has issued a report reflecting the work of a blue-ribbon panel that appeared about the time that APA began its public advocacy for prescription privileges. The report cites a number of criteria and content that are lacking in many current programs training practitioners to prescribe. The report addresses itself to medical education in the United States and does not even begin to speak to the issue of foreign medical graduates, who are overrepresented in the specialty of psychiatry. Indeed, the report suggests that we could accomplish a great deal in terms of quality improvement if we could simply teach all those who are currently prescribing or learning to prescribe how to do this part of their job.

Coming from the perspective of having $8+$ years of postbaccalaureate training-including the intensive 3-year pilot program of the Defense Department-Sammons is certainly entitled to a sense of confidence in his likely performance. However, our colleague's implicit contention that a much more limited and stand-alone module of learning units regarding prescriptive privileges is flawed in our view. We also doubt that it can be successfully delivered to psychologists in a workshop format when their mastery of premedical course material is varied. Given our extremely poor 
track record of success in endeavors such as trying to respecialize clinical psychologists as neuropsychologists in workshop formats, there is additional cause for concern. Despite this history, there is going to be no shortage of persons who wish to establish courses and content to teach prescribing; and it is surely not likely to be the curriculum Dr. Sammons has mastered. National College of Psychology, roll on!

But regardless of these more general reservations about feasibility, it is appropriate to deal with Sammons' issues as depicted above.

\section{NO BETTER, NO WORSE}

Sammons asserts that there is no reason to suppose that psychologists will be less effective in prescribing than others currently credentialed to do so. This logic runs counter to our societal ethic that safety-particularly in health matters-must be demonstrated and not presumed. At the same time, this conservative stance has perhaps rightly been thought to stifle innovation, change, and access to new ways of healing in endeavors such as development of new pharmaceuticals and utilization of physician extenders such as physician's assistants.

We also believe that Sammons' contention that psychologists are naturally more attuned to psychotherapeutic measures and will be less likely to overuse or rely on psychotropics is more wish than fact. Simply speaking, the current ethos in health care is to utilize the briefest treatment that works (or gives the appearance of working) and is balanced by a yield of greatest profit. Not only is pharmacotherapy undeniably cheaper than psychotherapy, but developments in mental health care are such that underwriters of care even want to decree strict protocols for the psychopharmacological treatment of depression and schizophrenia that are ordered solely by cost. In these circumstances even those long accustomed to being able to make complex decisions about "best" courses of prescribing strategy for patients will find themselves robbed of this professional prerogative and forced to follow song-card protocols that may not make sense except to corporate financial types counting ill-gotten gains "earned" from nothing less than the calculated imposition of suffering.

Do we as psychologists have the wish to come to this party?

\section{PALLIATIVE IS OK}

Sammons also thinks that our concern for the relative lack of curative medicines in the psychiatric formulary is curious. We cannot share that 
appraisal and only ask readers to compare psychopharmacology to other medical endeavors where drugs do other than to suppress symptoms. Let us - of course-allow that insulin-dependent diabetes is not quite subject to cure at this point and that medical replacement therapies only stave off the inevitable complications. The advance of medical science may yet knock off diseases that have hurt and killed mankind seemingly forever. Yet current developments in the biology of psychological disorders do not look anything like closure, and it may be premature for anyone to plan a trip to Scandinavia to accept a prize for conquering mental disorders anytime soon.

The brain is inherently complex and psychological/psychiatric disorders quite often sit in a much more complex matrix of causes and maintenance than does psoriasis. Why seek privileges to provide a medium of care that does not have a clear-cut improvement in our ability really to change patients for the better? In our experience, no small number of patients prefer to see a psychologist for the very reason that they are thus unlikely to be medicated.

We do not have a pie-in-the-sky ignorance of the very difficult and intractable nature of some psychological disorders, but we do own up to a perhaps (currently) politically incorrect confidence in psychological theory and method to do a power of good if given appropriate avenues of entry into health care. In this regard we feel that organized psychology has much more to gain by turning its efforts toward actions such as Capp v. Rank, which permit us to get into hospitals to show our skills to the (frequent) acclaim of nonpsychiatric physicians.

\section{MALPRACTICE? NO PROBLEM}

It is also Sammons' contention that we will not have a great deal to worry about with respect to malpractice, since only those psychologists who wish to prescribe will enter into the risk pool. The rates of complaints about psychologists failing to identify and refer medical problems in the APA data do not really relate to a psychologist provider community armed with prescription privileges. Of course, psychologists are not trained to identify medical problems, yet if they prescribe medicines, they will need to do so. Just because psychologists are as adept as psychiatrists in identifying somatoform disorders does not make them as good as physicians in identifying tardive dyskinesia, hypertension, or pulmonary edema. While there may indeed be no court award of damages for a psychologist for failing to identify medical problems to date, that is due in part to the fact that psychologists are not expected to identify medical problems. Get them 
prescribing and the expectation will come cheerfully along with a Greek chorus of "told-you-so" litigators.

While Sammons might feel that malpractice rates for psychiatrists are low, our research with Medical Risk Management Associates in Washington indicates that five-figure premiums per annum might strike psychology practitioners as trivial only in relation to, say, obstetricians these days. Thanks, but no thanks, we say.

It is also worth remembering that sexual misconduct with patientsonce rare as a charge in a court of law against psychologists-is still an isolated event. However, the small number of incidents captured by the press and celebrated at every recent APA meeting as a barometer of the integrity of therapists have extracted their toll. All psychologists have a premium added to the policies of the many for the transgressions of the few.

Also, given the strange events surrounding suits involving "the drug made me do it" claims for suicide and homicide cases, prescribers can expect with certainty that pharmaceutical manufacturers will effectively defend their products and that malpractice liability carriers will amortize their losses to policy holders. Put simply, for those colleagues like Sammons expecting justice and fair play for those bystanders not involved in legal fisticuffs, get your checkbook out.

\section{THE MOTIVATION}

There is no doubt, in our view, that the motivation to gain prescriptive privileges is economic and arguably proactive in the face of a changing health care delivery system. Managed care is knowingly strangling the delivery of mental health services, and the provider who will reach for a pad, rather than mud-wrestle a gatekeeper begging for permission for a few sessions, will have the upper hand or, at least, less risk for hypertension or stroke from massive incredulity. Psychologists may feel that they have an edge with prescription privileges, but when dealing with organizations determined to make profits at the cost of human suffering, the advantage is illusory. Organized psychology would serve the public better by using its resources to expose the fraud of savings in managed care and throw a highcandlepower light on managed care's attempt to carve out a middle-person's piece of the health care resource pie at provider and patient expense.

The use of rationales for prescriptive privileges involving "underserved" populations may be sensible from a tactical perspective and even accurate statistically. However, we think the use of rural mental health provider panel data to be opportunistic. The need for capable providers is 
also no less, for example, in the Alaskan Air Defense Command, but let us just be honest for a change and admit that these were prime tents for a camel's prescriptive privilege nose.

\section{THAT'S RIGHT: A JIHAD}

If we are indeed finally going to get honest with ourselves, let us admit that in going after the prescriptive privilege, we are implicitly gearing-up for a real test match with the other APA and, arguably, organized medicine. This may be the "smart" move, but our central thesis is that it may not be the best move for our profession. Nobody is likely to accuse the American Psychiatric Association of being overly collaborative or collegial, and its "mean as a snake" response to psychology on any front backed up with room-temperature intellectual collateral is counterproductive if not self-destructive, particularly when only $1 \%$ of a shrinking medical student share is electing psychiatry as a postgraduate concentration.

We have no special peacekeeping insights to share between professions. And indeed there is a decided lack of success in these healing endeavors around the world in these expedient days. However, we would like to make what some would see as the disturbing suggestion that common cause might yet be made against a more real and destructive corporate foe. It is frankly disappointing that both APA organizations have to waste money and time fighting each other as managed care predators run amok.

But as with all the other issues, there is a ready access to another "could" for our colleague. We would submit that in an all-out fight with organized medicine over access to prescription privileges, professional psychology would seem at first glance to be hopelessly outclassed from an economic and political point of view: until one recognizes that psychology may have in its corner some well-known multinational benefactors with names such as Burroughs Wellcome, Dista, and Roerig.

In sum, we do not agree with our colleague on a number of points. We do not believe that seeking prescriptive privileges is a wise move for organized psychology, but it must be said that a number of influential psychologists have been very active and successful in getting this issue tasked as a national priority for our APA. There does not seem to be much in the way of a discussion of whether or not this is wise or the will of the APA membership; and the raising of these "Should we?" questions usually prompts colleagues like Sammons to ask us essentially to prove to the null hypothesis that we should not do so. There is usually no arguing with science done in this fashion; but it may be that we are not in the business of science anymore, but in the science of business. 


\section{REFERENCES}

Adams, K. M., \& Bieliauskas, L. A. (1994). On perhaps becoming what you had previously despised: Psychologists as prescribers of medication. Journal of Clinical Psychology in Medical Settings, 1, 189-197

Capp v. Rank, No. S002524. Court of Appeals No. B020113, Los Angeles Superior Court C502929.

Sammons, M. T. (1994). Prescription privileges and psychology: A reply to Adams and Bieliauskas. Joumal of Clinical Psychology in Medical Settings, 1, 199-207. 